\title{
Gestión de políticas públicas en educación. Una aproximación desde lo municipal, nacional e internacional
}

\author{
Management of public policies in education. A municipal, national, and international approach
}

Gestão de políticas públicas na educação. Uma abordagem municipal, nacional e internacional

\author{
Miguel Bennasar ${ }^{1}$ \\ Instituto Superior de Formación Docente Salomé Ureña, \\ Santo Domingo - Santiago de los Caballeros, República Dominicana \\ (iD https://orcid.org/0000-0002-3856-0279 \\ miguelbennasar7884@gmail.com
}

DOI: https://doi.org/10.35622/j.rg.2022.01.006

Recibido: 22/11/2021 Aceptado: 23/02/2022 Publicado: 23/02/2022

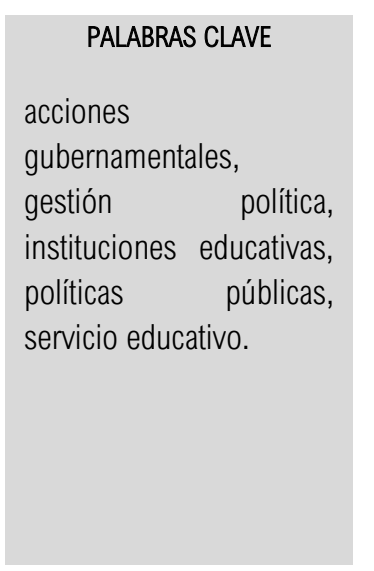

KEYWORDS
governmental actions,
political management,
educational institutions,
public policies,
educational service.

RESUMEN. El objetivo fundamental se orientó a estudiar la gestión de políticas públicas en educación en los contextos municipales, nacionales e internacionales en Venezuela. La metodología aplicada fue la investigación bibliográfica, que permitió una exploración exhaustiva de estudios e investigaciones, así como autores que han indagado los procesos formativos y las estrategias direccionales para un desarrollo armónico de este servicio fundamental hacia la comunidad. Dentro de los resultados, la principal limitación de las políticas públicas educativas del gobierno es la burocracia en la realización de los planes y proyectos, en el sentido de las posibles restricciones a la hora de diseñar, ejecutar y establecer un servicio que responda a la exigencia social. Como conclusión, el tipo de políticas se aplican en función de los objetivos y aspiraciones del gobierno y del tipo de ciudadano que se pretende formar, ello implica aplicar y ejecutar acciones que tiendan a mejorar las condiciones institucionales, la preparación de los docentes y la revisión curricular. Por ello se recomienda seguir ahondando en el tema de manera continua de la gestión política y las acciones gubernamentales.

ABSTRACT. The main objective was oriented to study the management of public policies in education in the municipal, national, and international contexts in Venezuela. The methodology applied was bibliographic research, which allowed an exhaustive exploration of studies and research and authors who have investigated the formative processes and directional strategies for a harmonious development of this fundamental service to the community. Among the results, the main limitation of the government's educational public policies is the bureaucracy in realizing plans and projects, in the sense of possible restrictions when designing, executing, and establishing a service that responds to social demands. In conclusion, the type of policies is applied according to the objectives and aspirations of the government and the kind of citizen to be educated, which

\footnotetext{
1 Postdoctor en el Programa: Políticas Públicas y Educación. Correspondencia: miguelbennasar7884@gmail.com
} 
implies using and executing actions that tend to improve institutional conditions, teacher preparation, and curricular revision. For this reason, it is recommended to continue delving into the subject of political management and governmental actions on an ongoing basis.

\section{PALAVRAS-CHAVE}

ações governamentais, gestão política, instituições educacionais, políticas públicas, serviço educacional.
RESUM0. 0 principal objetivo era estudar a gestão das políticas públicas em educação nos contextos municipal, nacional e internacional na Venezuela. A metodologia aplicada foi a pesquisa bibliográfica, que permitiu uma exploração exaustiva dos estudos e pesquisas, assim como autores que investigaram os processos formativos e as estratégias direcionais para um desenvolvimento harmonioso deste serviço fundamental para a comunidade. Entre os resultados, a principal limitação das políticas públicas educacionais do governo é a burocracia na realização de planos e projetos, no sentido de possíveis restrições na concepção, execução e estabelecimento de um serviço que responda às demandas sociais. Em conclusão, o tipo de políticas é aplicado de acordo com os objetivos e aspirações do governo e o tipo de cidadão que pretende educar, o que implica aplicar e executar ações que tendem a melhorar as condições institucionais, a preparação dos professores e a revisão curricular. Recomenda-se, portanto, que a questão da gestão política e das ações governamentais seja mais explorada de forma contínua.

\section{INTRODUCCIÓN}

Las políticas públicas se constituyen en orientaciones de servicios que atiendan al mayor número de ciudadanos de una sociedad, se trata de una posibilidad que se establece entre el gobernante y los gobernados para dirimir y establecer mecanismos de compensación en función de las diferentes problemáticas, que emergen del quehacer diario.

Las experiencias y vivencias que se han suscitado en Venezuela y Latinoamérica hacen reflexionar sobre los cambios profundos que en materia educativa se deben experimentar, entre ellos "... la democratización e inclusión en los sistemas y subsistemas escolares..." (Marín et al., 2020, p. 86), que sin duda alguna han impactado de manera directa a los sectores más vulnerables de la sociedad, poniendo en peligro su inclusión efectiva en los planes y programas de formación. Hoy se intentan desarrollar dentro de los nuevos programas curriculares educativos propuestas que van desde la visión municipal de la educación hasta la inserción en planes internacionales, que "... los retos y desafíos de la educación en todos sus niveles y modalidades, desde su rol transformador" (Antiche et al., 2021, p. 206).

En el caso de Venezuela, que es el contexto donde se desarrolla el presente estudio, las políticas públicas en materia educativa están orientadas a satisfacer a la mayoría de las personas en edad escolar. No obstante, a ello se han suscitado ciertas restricciones que han impedido su aplicación y desarrollo armónico como lo establecen tanto la Carta Magna como la Ley Orgánica de educación. En este orden de ideas Alija (2020) expone que:

Para lograr los más altos niveles de promoción y protección de los derechos humanos en el interior de los Estados, no basta con que estos introduzcan en su ordenamiento jurídico los estándares jurídicos internacionales en materia de derechos humanos. Si bien las medidas legislativas son una manera de dar cumplimiento a las obligaciones jurídicas internacionales convencionalmente establecidas, pocas veces son suficientes para asegurar que se garantiza en la práctica el pleno disfrute de los derechos humanos (pp. 641-642).

Siendo la educación un derecho humano básico para todas las personas que habitan un territorio, el desarrollo de acciones que se oriente hacia el establecimiento de las condiciones infraestructura y de servicios, es lo que 
priva para que las instituciones funcionen de manera adecuada. Esto permitiría, en el caso venezolano, incorporar a la mayor cantidad de personas en los diferentes niveles y modalidades del sistema educativo, lo que se revertiría en acciones de la propia comunidad hacia sus integrantes de manera positiva. "Así, las políticas formuladas durante este largo período involucraron una amplia gama de intercambios de información y aprendizaje político y gerencial favorecido, especialmente aquellos que utilizaron la construcción y afirmación de nuevas “ideas" para validarse" (Leite \& Sabourin, 2021, p. 510).

El trabajo se desarrolló basado en las experiencias que el autor ha realizado en los intercambios formativos en el programa de postdoctorado de la Universidad del Zulia, Venezuela, donde se discutieron a fondo la problemática de las políticas públicas educativas en los contextos municipal, nacional e internacional, como una aproximación a la realidad que describe el quehacer formativo en el país.

El propósito fundamente se orientó a estudiar la gestión de políticas públicas en educación en los contextos municipales, nacionales e internacionales en Venezuela, como alternativa metodológica la investigación bibliográfica, que permitió la realización de una exploración minuciosa de la temática, así como autores e investigaciones que han estudiado la educación y las estrategias direccionales para un desarrollo armónico de la educación como servicio fundamental hacia la comunidad.

\section{MÉTODO Y MATERIALES}

El presente estudio estuvo inserto en el paradigma cualitativo en el sentido de que dentro de las estrategias de análisis y de registros se realizaron descripciones y caracterizaciones de los hallazgos de acuerdo a las búsquedas que se realizaron en función de las aproximaciones hacia la Gestión de políticas públicas en educación en Venezuela en tres escenarios: municipal, nacional e internacional.

El tipo de investigación asumida fue la bibliográfica, pues se recopilaron informaciones de importantes bases de datos que abordaron el tema, así como de trabajos de investigación sobre temáticas similares. Este tipo de investigación considera todas las fuentes del saber para la obtención de informaciones relevantes y válidas de acuerdo al tema que se desarrolla y que son de interés para lograr los objetivos o propósitos planeados. Se constituye en sí misma en la revisión de producciones intelectuales que forman parte del estado del arte y de los estatutos epistemológicos que la caracterizan (Llumiquinga \& Vinueza, 2021).

\section{Nivel de la investigación}

El presente trabajo cuyo eje orientador son las políticas públicas educativas en Venezuela es de tipo descriptivo, explicativo-analítico, esto se justifica por las caracterizaciones que se derivaron de los hallazgos donde se especifican sus propiedades y justificaciones en el contexto formativo de acuerdo a las diferentes revisiones documentales realizadas (Arias, 2018).

Es de tipo explicativo-analítico en el sentido de que se precisan los argumentos de manera razonada de cómo se aplican las políticas públicas en el país en lo municipal, nacional e internacional, permitiendo realizar un recorrido interesante en datos e informaciones que han debatido esta temática. Cuando en investigaciones documentales se argumenta en razón de referentes teóricos, permite la consistencia de los hallazgos (Ortiz, 2020). 


\section{Diseño de la investigación}

El diseño que se adoptó es de tipo documental-no experimental, en el sentido de que las diferentes categorías asociadas a las políticas públicas en materia educativa fueron debatidas de acuerdo a las fuentes documentales utilizadas, por ello es que se considera una investigación bibliográfica (Bárcenas \& Preza, 2019).

Las unidades de estudios son todos los referentes documentales que tratan la temática de la educación como políticas públicas y que son referentes que se consideran en el presente estudio.

Los criterios de inclusión se explican en el sentido de que se consideraron artículos e investigaciones con datas no mayores a cinco (5) años de publicación, que hayan indagado sobre las políticas públicas educativas, y que sean publicaciones en revistas indexadas. No se incluyen los trabajos con datas mayores a 10 años, y las que no pertenezcan a publicaciones indexadas.

\section{RESULTADOS}

\section{Aproximaciones teóricas a las políticas públicas educativas}

Cuando se hace referencia a la condición epistemológica de las políticas públicas en materia educativa, las cuales se conciben como acciones dirigidas que tratan de satisfacer no solo demandas, sino los servicios formativos que tienen que ver con lo académico, social y de protección necesarios para un convivir sano y civilizado.

Una aproximación a su naturaleza es un ejercicio hermenéutico que procura constructo teórico que dé cuenta de las vicisitudes en estos ámbitos, que implica un análisis que tome en cuenta los aspectos epistemológicos en el contexto de una hermenéutica contemporánea, centrada en una ciudadanía que reclama una discusión pensada y responsable (Lozano, 2021).

Es por ello que cuando nos referimos a una aproximación a la naturaleza de las políticas públicas educativas, debemos estar conscientes que su impacto en el análisis debe considerar los aspectos que permiten una descripción razonada y justificada de esencia, es una “... Situación que genera desafíos y exigencias de diferente naturaleza, en términos de implementación de políticas públicas en todos los ámbitos..." (Díaz, 2020, p. 37).

Al tratar de descifrar lo que las políticas públicas educativas significan en un contexto determinado, implica razonar sobre los acontecimientos que las justifican y cuál es su propósito. Cuando se trata de políticas públicas se debe considerar la construcción interpretativa de las acciones hacia el bien común (Urbina, 2020).

Una primera aproximación parte de su descripción que se orienta a satisfacer los requerimientos formativos de una sociedad, su preparación para cumplir los diferentes roles que exige cada área de trabajo. Esta premisa surge de la interpretación etimológica del término, que invoca servicio, resolución de conflictos y aplicación de normas que viabilicen los propósitos educativos.

La educación es uno de los factores que más influye en el avance y progreso de personas y sociedades. La educación además de proveer conocimientos, enriquece la cultura, el espíritu, los valores y todo aquello que nos caracteriza como seres humanos. La educación siempre ha sido importante para el 
desarrollo, pero ha adquirido mayor relevancia en el mundo de hoy que vive profundas transformaciones, motivadas por el avance de la ciencia y sus aplicaciones (Manzanarez, 2020, p. 66).

Cuando se hace referencia a la construcción teórica referida a la a la naturaleza como esencia de las políticas públicas educativas, se debe interpretar que existe, en primer lugar, una justificación que dé cuenta de los aportes que en materia social o académica devienen de tales reflexiones, para de esta manera abrir espacios y escenarios de posibilidades que incluyan al mayor espectro de personas.

Las políticas públicas se han constituido como esos vehículos del crecimiento y del desarrollo; son estas políticas el mecanismo idóneo para que los gobiernos aborden temas de interés común, ya que son multidisciplinarias que abarcan aspectos que tienen que ver con la educación, salud, pobreza, economía entre otros (Espinoza, 2019, p. 39).

Si concebimos la posibilidad de una aproximación teoría de su naturaleza, en esta orientación, podemos asumirla como un conjunto de apreciaciones muy sistematizadas que se vinculan con lo social y lo educativo.

Si se piensa que para construir una política pública en particular se amerita objetivos bien definidos que sirvan de base para aproximarnos a la realidad que se ha pensado, se debe inferir que las mismas descansan en las posibilidades reales que la circunstancia social impone, y este es un insumo a considerar de manera seria.

En este contexto, el enfoque de la gobernanza pone el acento en la necesidad de un proceso de gobierno que logre construir un conocimiento colectivo causal basado en una racionalidad multidimensional con diversidad de actores, de enfoques, de recursos y de capacidades de adaptación. En otras palabras, apunta a la resignificación de la relación entre el gobierno y la ciudadanía, más allá del discurso optimista sobre las bondades de la participación ciudadana (Mayoral, 2020, p. 2).

La aproximación que teóricamente se pretende es concebida como la posibilidad de analizar las políticas públicas jerarquizándolas o dividiéndolas en subpartes que permitan una observación más sistematizada de los hechos, en este caso de las realidades educativas y sociales que se estudian.

Para Cardozo (2013):

Los gobiernos son considerados como los principales actores responsabilizados con las políticas públicas, debido a su privilegiada disposición de recursos y poder para ofrecer bienes, servicios y transferencias que resuelvan problemas públicos, si bien la participación de las organizaciones civiles y sociales y de los ciudadanos en general se considera altamente deseable (s/f).

Conociendo que las políticas públicas representan acciones de gobierno con objetivos marcados de interés público que promueven el desarrollo sustentable de una nación, el ámbito educativo es una pieza angular para la consecución de logros. En este sentido, las políticas públicas y la educación constituyen la vía esencial para promover y desarrollar el bienestar colectivo de la sociedad. Para ello se debe tomar en cuenta que la intencionalidad de estas radica en la necesidad e interés de que los servicios públicos de una nación sean eficientes, que, de manera interrelacionada, den respuestas a los problemas sociales para ofrecer una mejor calidad de vida a los ciudadanos. 
Es por ello que el abordaje de la educación como política pública de estado, a fin de reorientar los saberes hacia lo humano, sensible y pedagógico, busca promover en el sujeto una visión creativa, innovadora, crítica, reflexiva, transformadora y generadora de su aprendizaje para el desarrollo sostenible de una nación.

En este contexto, Urbina (2020) refiere que teorizar sobre las políticas públicas educativas, implica persuadir al pensamiento de que existen otras posibilidades y otros escenarios. Se vincula de esta manera que, desde la concepción teórica, el Estado en su responsabilidad de aplicar políticas públicas bien definida, debe considerar las experiencias que la realidad expone.

... siempre ha sido un servicio que brinda el Estado a la sociedad, se puede mencionar que es indispensable para el crecimiento político, económico y cultural de nuestro país, parte de la necesidad de mantener una tranquilidad social y convivencia armónica (Alonso \& Arenas, 2019, p. 3).

Es por ello que dentro del contexto de la práctica de una aproximación teórica de los estamentos públicos y educativos, se puede pensar en un constructo teórico cuyos elementos básicos se concentren en: a) La división efectiva de las políticas públicas en lo social y educativo, b) elaborar un esquema que permita una aproximación real y efectiva de las realidades de acuerdo a las responsabilidades que le son atribuidas, y c) la planificación de un programa de seguimiento o de supervisión que dé cuenta de los avances y de los logros o no de los objetivos previstos en una gestión determinada.

\section{Políticas públicas educativas en Venezuela en el ámbito municipal}

Las políticas públicas se constituyen en acciones dirigidas a satisfacer demandas sociales que imponen las comunidades y que los gobernantes están en la obligación de atender de forma eficaz y eficiente. En Venezuela por lo general se consideran como las diferentes actividades que desde las instituciones gubernamentales se realizan para satisfacer las demandas de las personas (Wilson, 2018). En este sentido las políticas públicas en materia de educación, el Estado y la sociedad civil deben construir se debe considerar las necesidades perentorias en cada sector, en el sentido de priorizar las mismas de acuerdo a los impactos en cada uno de ellos. Por lo tanto, se recomienda considerar la aplicación de acciones justas donde se aseguren los objetivos, deben considerar los recursos, así como las acciones de los funcionarios públicos vinculados con la implementación hacia la población y estratos vulnerables (Pineda, 2016).

En la construcción de políticas en materia educativa por ejemplo se deben considerar los elementos que permitan recuperar todas las infraestructuras y hacer una dotación que se ajuste a las demandas tecnológicas que marcan la pauta en la actualidad. El estado debe ser garante de esta política pública, pues se trata de la preparación de la sociedad en el devenir que se avecina.

La política pública ejecutada, que concreta los objetivos planteados y satisface los intereses, las necesidades y las expectativas a las que responde, es la que materializa la función pública. Esto no es fácil, pues ni el Estado ni la sociedad civil son entes unitarios y homogéneos; por el contrario, son fragmentados y heterogéneos. Por esta razón, ni uno ni otra pueden ejercer siempre el control absoluto sobre la acción institucional del Estado o de la sociedad civil, lo que hace relativa la manera como se concreta la función pública. Esta se ubica en el dominio de las pugnas por la hegemonía y se ve afectada por las tensiones generadas por los cambios en las correlaciones de fuerzas sociales (Pulido, 2017, p. 20). 
Es por ello que cualquier proyecto educativo debe tener como norte la formación de un ciudadano ejemplar, 0 mejor dicho la consolidación de un proyecto nacional que involucre a los sectores de todos los niveles, sin ningún tipo de discriminación, y en ello precisamente el Estado tiene una responsabilidad particular. Esta apreciación sin lugar a dudas es aplicable en lo social y el Estado como garante de la aplicación estrategias para la construcción de una mejor sociedad, debe considerar desde estas aristas, métodos inclusivos para que los servicios sean a favor de las grandes mayorías.

El Estado venezolano a través de sus políticas públicas manifiesta como objetivo alcanzar la justicia social basada en la inclusión social, para disfrutar los derechos, disminuyendo las inequidades y priorizando necesidades sociales. Asimismo, lograr el bien común es un valor preeminente; los valores culturales, la interculturalidad, el deporte y recreación. Ética del trabajo, participación ciudadana, corresponsabilidad educativa, actualización y tecnología, identidad nacional y respeto a corrientes de pensamiento, las cuales son políticas emprendidas por el Estado en materia educativa (Núñez et al., 2017 sp.).

En este sentido, la Constitución de la República Bolivariana de Venezuela le da a la municipalización de la educación una connotación que se basa en las propuestas políticas que se derivan de este instrumento jurídico y que ha cobrado cierta legitimidad por el acercamiento que se ha experimentado entre las instituciones educativas y la sociedad. En efecto, “... El Municipio se configura como la instancia participativa por excelencia de la vida ciudadana, en sus diversas manifestaciones entre las cuales resalta la experiencia educativa..." (Morales \& Núñez, 2006, p. 401). Por tanto, el sendero posible en el contexto de las políticas públicas en la actualidad a nivel local y municipal es procurar mediante estas políticas propiciar un servicio educativo que desarrolle planes y programas hacia el logro de la inclusión efectiva de todos los sectores a los servicios educativos, y esta es una deuda social por satisfacer.

Direccionar las políticas públicas de la educación en el ámbito municipal en la actualidad, constituye centro de atención en disímiles y variados contrastes. "Aunque Venezuela no cuenta con pruebas estandarizadas de medición de los aprendizajes, las graves condiciones del entorno socioeducativo presagian un bajo rendimiento de los estudiantes venezolanos" (Medina, 2021, p. s/p). Se requiere entonces de un abordaje que trascienda los enfoques que han excluido a un vasto sector de la población, y que no se puede seguir eludiendo.

Los obstáculos que hoy impiden aplicar políticas públicas en materia educativa han sido vacuos, pues se trata de realizar acciones, que permitan capturar la realidad desde una relación dialéctica entre sus protagonistas. Es por ello que se debe realizar una interpretación de la realidad este por encima de los supuestos e hipótesis que ha obstaculizado el desarrollo efectivo del país.

Pues los derechos sociales inherentes a la persona como “...la educación, así como las obligaciones que contraen los estados para la plena realización del mismo, es fácil observar que algunos son programáticos o de desarrollo progresivo, (...), mientras que otras son de cumplimiento inmediato (...). (Bolívar, 2010, p. 16). Donde resaltan la inclusión y la participación. Y esta debe ser la ruta de toda política pública, más aún si se trata del área educativa por su importancia para el desarrollo y la emancipación. 


\section{Consideraciones de las políticas públicas en el contexto nacional venezolano}

El establecimiento de políticas públicas en materia educativa en el ámbito venezolano parte de la premisa de que las condiciones institucionales son las adecuadas, sin embargo, la realidad es otra. En este sentido se trata de dotar de los insumos mínimos necesarios a estas instituciones para que funcionen adecuadamente y presten un servicio de calidad a la sociedad. En esto el Estado tiene una responsabilidad superior.

Reyes y Rivera (2018) señalan:

El aspecto socio-cultural se refiere a la identidad, conciencia y sentido de pertenencia a una comunidad política y a las prácticas individuales y colectivas para ejercer derechos y cumplir las responsabilidades ciudadanas. El aspecto institucional tiene como finalidad proteger los derechos y regular las responsabilidades que se encuentran reconocidas en las constituciones políticas nacionales y en los tratados de la órbita de Naciones Unidas (principalmente el Pacto Internacional de Derechos Civiles y Políticos y el Pacto Internacional de Derechos Económicos, Sociales y Culturales) (s/p).

El ejercicio hermenéutico que implica una apuesta verdadera en materia educativa, debe considerar los aspectos y acciones que se han aplicado y que eventualmente no han producido los resultados esperados: una mejor educación, una sociedad conocedora de sus deberes y derechos y un Estado con plena conciencia de su responsabilidad. Esto dará cuenta de manera inequívoca de los errores y de las políticas aplicadas que incluyen la coyuntura actual. De tal manera que en lo atinente al Estado se debe considerar los ministerios, planes y programas en su totalidad; en materia educativa revisar adecuadamente las políticas aplicadas por los Ministerios del área y en lo social establecer vínculos con la comunidad para apreciar de manera objetiva el impacto de las políticas públicas aplicadas en los procesos de construcción, deconstrucción y reconstrucción. Este ejercicio dará luces para próximas acciones.

Los procesos que se desarrollan en la aplicación de políticas públicas en materia educativa son escenarios que permiten explorar ideas, evaluar escenarios y en el área formativa tiene una pertinencia especial. "Este hallazgo es relevante debido a que el componente epistemológico es el fundamento de todo el proceso educativo tanto en lo pedagógico, como lo didáctico y es donde el docente muestra que es experto en su área disciplinar" (Hernandez et al., 2019, p. 132).

Es por ello que en el ámbito social tiene una pertinencia en la coyuntura actual muy particular, se trata de establecer las bases de una nueva convivencia, con reglas novedosas que den cuenta de otras posibilidades, ya que la visión que se tiene de la malla donde la cotidianidad forma parte del ser, debe ser desmontada y reedificada. “... Se busca que trascienda la existencia, que la vida recobre otro sentido, que se empoderen los sueños y las esperanzas desde otra realidad distinta donde el individuo se encontraba (Rincón, 2015, s/p). En ello el Estado como poder político puede desarrollar acciones que permitan transitar otros escenarios y donde la sociedad juegue un papel protagónico de acuerdo a los cambios que se ejecuten o surjan de esta práctica.

Es así que la educación, en lo social y lo estatal, son protagonistas de posibilidades que van más allá de las previsiones o programas, Ellos trascienden los senderos que se han trazado, por ello cuando se habla de posibilidades educativas, hay que considerar las potencialidades que emergen de cada uno de los sectores involucrados. 
En términos generales, la política pública se refiere al vínculo entre el Estado y la sociedad que, en realidad, toma cuerpo en la relación entre gobernantes y gobernados, extendiéndose al ámbito de la cultura, en función de los intereses y necesidades concretas que convocan a los distintos grupos humanos a la acción política. Por lo general, la política pública se aborda desde visiones técnicas e instrumentales preocupadas por las metodologías y procedimientos para su elaboración, por los diagnósticos, fases, definición y cumplimiento de objetivos y metas, rutas, cronogramas, recomendaciones, con criterios de evaluación por eficacia, eficiencia y efectividad (Pulido, 2017, p. 16).

Evidentemente que la responsabilidad gubernamental en esta coyuntura es de primer orden y sus acciones deben estar en consonancia con los requerimientos sociales y educativos por añadidura, pues se debe considerar los desafíos que la coyuntura actual enfrenta, las prerrogativas que devienen en el transcurso de la historia por venir.

Cuando se hace referencia a la responsabilidad del Estado, se interpreta una acción de cuestionamiento a como se desarrollan las políticas públicas en cualquiera de sus acepciones: sociales, nacionales, educativas y de cualquier otra índole. Se trata de una acción que da cuenta del estado del arte de una situación específica y como gravita en cada uno de los sectores involucrados.

Toda propuesta educativa debe desmontar los estados actuales, examinar, evaluar y supervisar los acontecimientos desde la óptica donde ocurren los hechos. Interpretar de manera adecuada todos los elementos constitutivos permite una observación detallada par precisar las acciones que se realizaron, sus efectos, el logro o no de los objetivos propuestos, hasta diagnosticar la adecuabilidad o no de tales políticas públicas.

Las políticas públicas en lo educativo y en lo social, deben aperturar espacios para que la sociedad desarrolle sus potencialidades y pueda auto sustentarse, es decir que no sea dependiente exclusivamente del Estado, porque este tipo de acciones han sido un erráticas. Los pueblos deben construir sus posibilidades y el Estado potenciarlas.

\section{Una aproximación de las políticas públicas en el ámbito regional latinoamericano}

A nivel internacional, específicamente en América Latina, la ruta que se debe recorrer debe considerar la promoción efectiva de políticas públicas educativas que permitan al mayor número de personas a este servicio de vital importancia. Muchos son los países que han desarrollado e implementado acciones en este sentido, que dan cuenta del avance y desarrollo hacia la no discriminación en estas naciones.

La región de América Latina tiene gobiernos que están muy calcados en su estructura estatal, y cuando presentan declives al momento de ejercer sus funciones pasan por un momento de restructuración en la que prácticamente se engloba toda la región (Estupiñan, 2020, p. 6).

La UNESCO (2015) sostiene: "Los niveles cada vez más altos de complejidad social y económica presentan diversos desafíos para la formulación de políticas en el mundo globalizado de hoy” (p.10).

Los organismos internacionales tienen una participación importante en el financiamiento de las políticas públicas puesto que, los aportes de recursos les facilitan su intervención en temas sensibles de los países de Latino América (Rafael Marte, 2017). 
Se trata de una realidad que ha persistido por mucho tiempo pero que ha motivado a los administradores y gerentes institucionales a repensar las políticas educativas que se aplican y que muchas veces se desvían y afectan a un número significativo de personas.

Al respecto, Paya (2020) sostiene:

Atender la diversidad implica asegurar la igualdad de oportunidades y luchas contra la discriminación en la promoción de la equidad. Algunos países tienen una visión más comprensiva de la educación inclusiva (Argentina, Brasil, Ecuador, Honduras, Paraguay, Uruguay, Perú o Venezuela), otros carecen de planteamientos claros prioridades en educación inclusiva (Bolivia, Guatemala), o bien la vinculan a la educación especial y la atención a alumnos con necesidades educativas especiales (El Salvador) 0 incluso combinan una visión ampliada de la educación inclusiva con políticas de atención a las necesidades educativas especiales (Nicaragua) (p. 129).

La justeza de la aplicación de políticas educativas que incluyan las personas como derecho humano no debe ser un capricho, sino un proyecto de todos los gobiernos del mundo, pues si la persona esta educada los procesos formativos han funcionado y sin lugar a dudas se tendrá un contingente útil para sí mismo y para la sociedad (Jiménez \& Ortega, 2018). Esta debe ser la tendencia en toda la región y a nivel planetario: más y mejor educación como política pública emergente.

Se trata de un cambio de conducta que debe partir desde las esferas altas de los gobiernos como símbolo inequívoco de progreso, esta debe ser la ruta a transitar a través de la aplicación de políticas públicas en materia educativa para alcanzar estadios de progreso y desarrollo de manera sostenible. En este caso se debe incluir a todos los sectores de la sociedad, con preeminencia en la coyuntura actual donde se debaten mecanismos y acciones que permitan alcanzar los propósitos esenciales de toda sociedad: vivir en armonía y procurar satisfacer las necesidades más urgentes como principio de convivencia.

\section{¿Cuáles serían los atisbos para el diseño de una posible propuesta?}

Construir políticas públicas que involucren los aspectos sociales y educativos por parte del estado, dentro de los posibles atisbos que subyacen en las reflexiones teóricas y epistemológicas, implica realizar esfuerzos comunes que permitan, en primer lugar, encontrar encrucijadas comunes para aunar esfuerzos y posibilitar acciones que beneficien y satisfagan las demandas de las mayorías. Una posibilidad que puede explorarse en el contexto de las políticas públicas tiene que ver con los insumos o requerimientos necesarios para emprender un ejercicio expedito, pues “... cuando se trata de una construcción fundamentalmente interpretativa, en este tipo de teoría no se descarta su capacidad explicativa y predictiva” (Arias, 2018, p. 8).

Otra conjetura que se puede discutir tiene que ver con las concepciones reales que desde la visión del Estado se tienen de las políticas públicas, además de las prerrogativas programáticas que se construyen, se deben considerar a quienes van dirigidas las mismas, de tal manera que dependiendo de del aspecto utilitario, se pueden inferir las acciones pertinentes para su ejecución. En sentido lo expuesto por Corzo (2013) se orienta en la perspectiva de lo epistemológico, que es una de las aristas que evidentemente se deben apostar convirtiéndose en la apotema como acción inmediata en el quehacer gubernamental. Es decir, buscar las vías 
que mejores resultados puedan aportar en la orientación de resolver los nudos coyunturales en lo social y educativo.

... se puede concluir que las políticas públicas son acciones que permiten un mejor desempeño gubernamental, tanto al interior como al exterior del aparato público, a partir de cuatro supuestos: el interés público, la racionalidad, la efectividad y la inclusión. Tales premisas se logran a través del uso racional de los recursos públicos, la focalización de la gestión gubernamental a problemas públicos acotados y la incorporación de la participación ciudadana (Corzo, 2013, s/p).

El autor expone un atisbo que tiene que ver con el uso correcto de los recursos, es decir, hacer uso adecuado de los insumos que se le asigna a cada entidad gubernamental para que se administre en función de las demandas que devienen del devenir social. Esta conjetura que se expresa en el entendido de que a partir de la consideración de las políticas públicas, que de acuerdo con Lobelle (2017) se concibe como la diversidad de acciones que se implementan de acuerdo los planes y programas en favor de la comunidad. Significa desde esta óptica que es posible implementar planes y programas de manera versátil en función de los grandes objetivos sociales y educativos.

Por esta razón han surgido en los últimos años numerosas publicaciones tendientes a ayudar a los interesados a crear sistemas de monitoreo y evaluación adecuados, que permitan un mayor flujo de información de cara a futuras decisiones de política; aunque las sugerencias no siempre coinciden, hay elementos que son comunes y sobre los cuales vale la pena hacer énfasis (Dorado, 2010, s/p).

De tal manera que la consideración de los insumos o de los presupuestos como elementos que facilitan la aplicación de políticas públicas en función del colectivo, significa un atisbo importante que se debe analizar y considerar para futuros proyectos dentro de los contextos que se analizan.

Significa que, a pesar de que los gobiernos tienen como responsabilidad la aplicación de políticas públicas ajustadas a los requerimientos sociales y en este caso en lo educativo, muchas veces no son asumidas con la vehemencia que las circunstancias demandan, produciendo un atisbo adicional que consiste en la responsabilidad y seriedad de quienes ejercen y aplican tales políticas en el entendido del compromiso que ello refiere.

En lo educativo en el análisis de los atisbos como posibilidades para la ejecución de políticas públicas asertivas, se vislumbras panoramas que ameritan una revisión particular, esto dentro de algunas líneas por los resultados que muchas veces denuncian algunas fallas en cuanto a su implementación. Esto implica que las instituciones, así como sus autoridades deben asumir los roles de manera efectiva, pues no se trata de una función sin resonancia social pues los académicos (docentes y estudiantes) requieren de visiones claras y de propósitos plausibles.

Actualmente las políticas públicas se han desdibujado como concepto a consecuencia de la incapacidad de los círculos académicos y de los funcionarios por crear un consenso y definir qué son, qué hacen y cómo funcionan. Así mismo, el estudio e implementación de la política contiene vacíos académicos y técnicos, resultado del poco tiempo que lleva su utilización y los altos grados de empirismo con que se ejecutan (Arroyave, 2011, p. 96). 
Esta consideración inserta dentro de los roles de quienes ejecutan acciones gubernamentales dentro y fuera de las instituciones educativas están más que en la responsabilidad en la obligación de que sus acciones deben estar en consonancia con los principios administrativos de recursos de una comunidad o una institución, ese debe ser el norte y la consideración ulterior.

Es por ello que cuando se habla de los posibles atisbos en el contexto de las políticas públicas, se pueden enumerar un sinnúmero de conjeturas o de posibles acciones que eventualmente explican la naturaleza de las mismas. No obstante, a ello no se pueden realizar afirmaciones absolutas porque quienes ejecutan tales políticas son personas, y eso le dé un grado de subjetividad que pueden decantar en algunas limitaciones como: la falta de presupuesto, ausencia de planes y programas sociales o educativos, falta de coherencia entre lo planificado y los propósitos políticos.

Dentro de estas realidades lo que sí se puede asegurar que, si quienes ejercen los roles para la ejecución de las políticas públicas en una sociedad y coyuntura determinada, tienen la responsabilidad de viabilizar y buscar caminos cuyos atisbos no sean la excusa para no cumplir las responsabilidades inherentes a sus funciones. Se trata de un nivel de conciencia que va más allá de una simple reflexión teórica, pues bajo su manto se cubre las múltiples necesidades y esperanzas de quienes confían en sus gobernantes o directivos.

\section{CONCLUSIONES}

Los hallazgos muestran que las principales limitaciones de las políticas públicas educativas del gobierno es la excesiva burocracia en la realización de los planes y proyectos, en el sentido de las posibles restricciones a la hora de diseñar, ejecutar y establecer un servicio que responda a la exigencia social.

A nivel municipal se percibe que las orientaciones y ejecuciones de los planes de gobierno en materia educativa no cuentan con los recursos necesarios para su ejecución efectiva, aunque existe una relación estrecha entre los ciudadanos y las instituciones educativas.

En las diferentes localidades donde existen instituciones educativas, muchas de las políticas que se aplican están en franca contradicción con las necesidades básicas de la sociedad pues continúan no satisfechas. De ahí la importancia de que las obras gubernamentales estén en sintonía con el sentir de todas las personas atacando las posibles fallas que afectan las acciones comunitarias.

Las referencias que se realizan de las políticas educativas, se orientan hacia la condición social de los impactos que se generan, producto de las acciones acometidas y aplicadas por las entidades gubernamentales. Es una forma de gobierno con sensibilidad en las comunidades, en los estratos de diferentes clases para que sean consideradas dentro de las políticas que servirán para construir espacios de convivencia adecuados.

La metodología empleada en el presente trabajo sirvió de guía para la exploración de ideas, documentos e investigaciones que tratan el tema de las políticas públicas gubernamentales en los diferentes ámbitos, encontrándose mayormente que, dentro de los esquemas legales y constitucionales, existe una vía normativa para la ejecución de los planes que en esta materia se pueden aplicar. Hay una perspectiva positiva que muchas veces no cuentan con la ejecución efectiva de las mismas. Esta realidad no coloca en minusvalía el significado 
y practica de las políticas que se aplican en las instituciones de formación, pero abre un compás de análisis de estas realidades, cuyos efectos son negativos para lo sociedad, es lo que se debe evitar.

El proceso teórico de aproximación a las políticas públicas educativas, además de considerar las realidades y las visiones que se derivan de su apreciación epistemológica, permite edificar, desde los cimientos de las realidades o de los insumos particulares, una apuesta por el bienestar en lo social y educativo de manera convincente. Un conjunto de actividades que beneficien a la mayor cantidad de personas en edad escolar, interlocución entre los Ministerios del ramo y las diferentes autoridades locales, y ejecución de programas que retroalimenten las acciones institucionales. Son consensos que se deben poner en prácticas para que las políticas educativas tengan un soporte e importancia ministerial y gubernamental.

Para ello, debe contar con actores educativos comprometidos con elevar la calidad educativa en su más alto nivel; sin embargo, lobre todo, desarrollar una concepción del sujeto desde la razón y lo sensible, entendiendo que la educación es un proceso humano de formación continua que promueve conocimientos, principios y valores por y para la vida. He aquí el docente como elemento vital y comprometido desde sus funciones que despierte en el educando el aprender a enseñar, a aprender, realizando combinaciones de experiencias y saberes de lo cotidiano y lo académico, donde se incorpore lo afectivo, lo emocional, lo creativo, como ingredientes medulares y promotores de su condición humana, relacionado con el entorno social.

Toda aproximación de una realidad implica revisar diferentes vertientes, es un recomenzar, con la diferencia de que existe una experiencia que puede servir de guía a los nuevos estatutos en materia de políticas públicas.

Tanto las políticas públicas educativas como la gestión pública de gobierno pertenecen al ámbito del poder ejecutivo en la toma de decisiones, que son los garantes en primera instancia en la puesta en práctica de los planes y programas en cada una de las instituciones nacionales.

Este tipo de políticas en su conjunto se aplican en función de los objetivos y aspiraciones del gobierno y del tipo de ciudadano que se pretende formar, ello implica aplicar y ejecutar acciones que tiendan a mejorar las condiciones institucionales, la preparación de los docentes y la revisión curricular anualmente para apreciar los impactos en los procesos formativos.

Las políticas públicas educativas son programas que ejecutan por orientaciones del ejecutivo con impacto nacional e internacional. Su naturaleza da un horizonte que aborda dentro de los esquemas y de sus planes, las siguientes orientaciones:

Como colofón se puede argumentar que las políticas públicas educativas, a partir de las reflexiones esgrimidas, son de gran importancia para cualquier sociedad, en virtud de su orientación en cuanto a lo utilitario en el propósito de insertar a los egresados en las actividades laborales de manera efectiva.

\section{Conflicto de intereses / Competing interests:}

$\mathrm{El}$ autor declara que no incurre en conflictos de intereses.

Rol de los autores / Authors Roles:

No aplica. 
Fuentes de financiamiento / Funding:

Los autores declaran que no recibió un fondo específico para esta investigación.

Aspectos éticos / legales; Ethics / legals:

El autor declara no haber incurrido en aspectos antiéticos, ni haber omitido aspectos legales en la realización de la investigación.

\section{REFERENCIAS}

Alija, R. (2020). La necesidad de transversalizar los derechos humanos en las políticas públicas para hacer frente a las crisis: una aproximación desde el derecho internacional de los derechos humanos. Revista Direitos Sociais e Políticas Públicas (UNIFAFIBE), 8(2), 640. https://doi.org/10.25245/rdspp.v8i2.831

Alonso, J., \& Arenas, R. (2019). Análisis jurídico de la naturaleza de la seguridad pública en México. Revista Dilemas Contemporáneos: Educación, Política y Valores, 4(3). dilemascontemporaneoseducacionpoliticayvalores

Antiche, J., Piñero, M., Vanga, M., Sáenz, J., \& Lucas, C. (2021). Acción pedagógica para la innovación en la educación técnica industrial: Una aproximación etnográfica. Revista EDUCARE - UPEL-IPB - Segunda Nueva Etapa 2.0, 25(1), 201-223. https://doi.org/10.46498/reduipb.v25i1.1460

Arias, F. (2018). Diferencia entre teoría, aproximación teórica, constructo y modelo teórico. Sciencie, 10(2). https://revistas.upel.edu.ve/index.php/actividadfisicayciencias/article/view/7361

Arroyave, A. (2011). Las políticas públicas en Colombia. Insuficiencias y desafíos [Universidad Nacional de Colombia]. https://repositorio.unal.edu.co/handle/unal/42681

Bárcenas, K., \& Preza, N. (2019). Desafíos de la etnografía digital en el trabajo de campo onlife. Virtualis. Revista de cultura digital, 10(18). https://doi.org/10.2123/virtualis.v10i18.287

Bolívar, L. (2010). El derecho a la educación. Revista Instituto Interamericano de Derechos Humanos, 52, 191-212. https://www.corteidh.or.cr/tablas/r25566.pdf

Cardozo, M. (2013). Políticas públicas: los debates de su análisis y evaluación. Andamios, 10(21). http://www.scielo.org.mx/scielo.php?script=sci_arttext\&pid=\$1870-00632013000100003

Corzo, J. (2013). ¿Qué son las Políticas Públicas? IEXE Universidad.

Díaz, M. (2020). Propuesta de medidas jurídicas para la reinserción y permanencia laboral del adulto mayor en las políticas públicas peruanas. IUS: Revista de investigación de la Facultad de Derecho, 9(2), 18-39. https://doi.org/10.35383/ius-usat.v9i2.449

Dorado, (2010). P. Políticas públicas hoy (Vol. 2$)$ 2) https://colaboracion.dnp.gov.co/CDT/Sinergia/Documentos/Boletin_Politica_Publica_Hoy_02.pdf

Espinoza, A. (2019). Estudio comparativo de las políticas públicas en competitividad a nivel centroamericano. Innovare: Revista de ciencia y tecnología, 7(1), 38-57. https://doi.org/10.5377/innovare.v7i1.7570

Estupiñan, G. (2020). Políticas públicas para el desarrollo local de la parroquia de Borbón, en la provincia de Esmeraldas [Universidad de Guayaquil]. http://repositorio.ug.edu.ec/handle/redug/50685

Hernandez, Y., Hernandez, A., \& Navarro, M. (2019). Deconstrucción y reconstrucción de las practicas pedagógicas en torno a la formación por competencias en el área de ciencias naturales grado cuarto de básica primaria en las instituciones educativas Luis Gabriel Castro Sede San Pedro y el Instituto Técnic [Universidad Simón Bolívar]. https://hdl.handle.net/20.500.12442/3929\%09

Jiménez, M., \& Ortega, P. (2018). Referentes sobre inclusión educativa para personas con discapacidad: líneas para pensar su potencial en el ámbito escolar. Civilizar, 18(34), 85-104. https://doi.org/10.22518/usergioa/jour/ccsh/2018.1/a06 
Leite, S., \& Sabourin, E. (2021). Introdução à seção temática: Entre continuidades e descontinuidades das políticas públicas para a agricultura brasileira. Estudos Sociedade e Agricultura, 29(3). https://doi.org/10.36920/esa-v29n31

Llumiquinga, E., \& Vinueza, N. (2021). Investigación bibliográfica sobre el tratamiento fisioterapéutico temprano en pacientes oncológicos post mastectomía [Universidad Central de Ecuador]. http://www.dspace.uce.edu.ec/handle/25000/23735

Lobelle, G. (2017). Políticas públicas sociales: apuntes y reflexiones. Alcance, 6(14). http://scielo.sld.cu/scielo.php?script=sci_arttext\&pid=\$2411-99702017000300006

Lozano, K. (2021). Políticas públicas para una economía creativa en Jalisco y el área metropolitana de Guadalajara. región y sociedad, 33, e1375. https://doi.org/10.22198/rys2021/33/1375

Manzanarez, B. (2020). Políticas Públicas en la Educación Superior Virtual Nicaragüense. Raíces: Revista Nicaragüense de Antropología, 64-71. https://doi.org/10.5377/raices.v3i7.9697

Marín, M., Hinojosa, G., \& Tomalá, A. (2020). Factibilidad de la carrera de educación básica general en la Universidad Laica Vicente Rocafuerte de Guayaquil. Yachana Revista Científica, 9(3), 85-92. http://revistas.ulvr.edu.ec/index.php/yachana/article/view/681

Mayoral, 0. (2020). Participación ciudadana y politicas públicas culturales. Aproximación de lo municipal a partir del primer año de trabajo de las administraciones Hermosillo 2015-2018 y 2018-2021 [El Colegio de Sonora]. https://repositorio.colson.edu.mx/handle/2012/44410

Medina, V. (2021). El sistema educativo venezolano en terapia intensiva. Debates IESA. http://www.debatesiesa.com/elsistema-educativo-venezolano-en-terapia-intensiva/

Morales, E., \& Núñez, I. (2006). La municipalización de la educación superior en la República Bolivariana de Venezuela. Revista Venezolana de Ciencias Sociales, 10(2), 393-403. https://www.redalyc.org/pdf/309/30910207.pdf

Núñez, I., Morales, E., \& Díaz, I. (2017). El replanteamiento de las políticas educativas en Venezuela. Gaceta Laboral, 13(3). http://ve.scielo.org/scielo.php?script=sci_arttext\&pid=\$1315-85972007000300004

Ortiz, R. (2020). Análisis métrico de la producción científica sobre COVID-19 en SCOPUS. Revista Cubana de Información en Ciencias de la Salud, 31(3). http://scielo.sld.cu/scielo.php?script=sci_arttext\&pid=S2307-21132020000300002

Paya, A. (2020). Políticas de educación inclusiva en América Latina. Propuestas, realidades y retos de futuro. Revista de educación inclusiva, 3(2). https://revistaeducacioninclusiva.es/index.php/REl/article/view/209

Pineda, Y. (2016). Aprender a construir y reconstruir desde una mirada investigativa en los espacios de educación superior. Revista Ciencias Humanas, 13(1). https://doi.org/10.21500/01235826.2965

Pulido, 0. (2017). Política pública y política educativa: una reflexión sobre el contexto. Revista Educación y Ciudad, 33, 13-28. https://doi.org/10.36737/01230425.v0.n33.2017.1641

Rafael Marte. (2017). Importancia de la gestión de políticas educativas y la influencia que tienen los organismo internacionales (OCDE, Banco Mundial, UNESCO y PREAL) en las reformas de los sistemas educativos. Revista Atlante: Cuadernos de Educación y Desarrollo. https://www.eumed.net/rev/atlante/2017/04/politicas.html

Reyes, 0., \& Rivera, J. (2018). Construcción de ciudadanía: la educación desde la infancia encaminada a la inclusión social. Tla-melaua, 12(44), 52-71. http://www.scielo.org.mx/scielo.php?pid=S1870$69162018000100052 \&$ script=sci_abstract

Rincón, A. (2015). La reconstrucción del tejido social y la persona: filosofía de la educación. Actas asociacion Latinoamericana de filosofia y educacion, 3. http://filosofiaeducacion.org/actas/index.php/act/article/view/146 
UNESCO. (2015). Replantear la educación ¿hacia un bien común mundial? http://hdl.handle.net/20.500.12799/3760\%0A

Urbina, M. (2020). Las Políticas Públicas venezolanas en materia de género: Tradición vs demandas sociales. Encuentros. Revista De Ciencias Humanas, Teoría Social Y Pensamiento Crítico, 11, 41-56. https://doi.org/10.5281/zenodo.3693024

Wilson, J. (2018). ¿Qué son y para qué sirven las políticas públicas? Revista IN IURE, 2(8), 30-41. https://revistaelectronica.unlar.edu.ar/index.php/iniure/article/view/492 